\title{
Language and Culture Appropriate Approaches Needed to Improve Health Education to Reduce Disparities among Korean American Immigrants Living in Los
} Angeles

\author{
Muhammad Shahid ${ }^{1}$, Austin Yeon1, Eunho Cho ${ }^{1,2}$, Jooeun Bae ${ }^{1,2}$, Rebekah J. Park ${ }^{1}$, Jayoung Kim ${ }^{1,2,3,4, ~ đ I ~}$ \\ ${ }^{1}$ Departments of Surgery and Biomedical Sciences, Cedars-Sinai Medical Center, Los Angeles, CA, USA; \\ ${ }^{2}$ University of California Los Angeles, CA, USA; \\ ${ }^{3}$ Samuel Oschin Comprehensive Cancer Institute, Cedars-Sinai Medical Center, Los Angeles, CA, USA; \\ ${ }^{4}$ Department of Urology, Ga Cheon University College of Medicine, Incheon, Republic of Korea
}

\begin{abstract}
This study aimed to determine the disparities associated with health awareness and access to health education among Korean American residents living in the Los Angeles area. In particular, this research focused on gathering data on how Korean American immigrants perceive health information and what major barriers prevent them from gaining access to healthcare. In total, 421 Korean Americans living in Los Angeles participated in this study. The constructed 13-item questionnaires were provided in a pen and paper or online version in Korean or English. The quantitatively evaluated data revealed the status of exercise, smoking, access to healthcare, health interest, alcoholic consumption et al. in female and male subjects. Our study found that older age groups chose the paper survey option rather than the online version. They also preferred the Korean-translated survey over the English one. These findings suggest that careful consideration on language and format is required when assessing healthcare access in older generation Korean Americans. The major health concern of the paper survey group was also cancer prevention.
\end{abstract}

Keywords: Los Angeles; Korean American immigrants; health education; equity; healthcare

\section{Introduction}

As the United States becomes more linguistically and culturally diverse, there is a greater need for effective health communication interventions that target diverse and the most vulnerable populations (Barrera et al., 2013). Southern California, particularly Los Angeles and the Orange County area, has the highest concentrated Korean population in the U.S.; making up 25\% of all Koreans residing in America. A majority of Koreans are concentrated around the Koreatown area. Koreatown is also the most densely populated district, by population, in LA county, with an average of 42,611 people per square mile. However, research activities with special efforts on collecting health information on ethnic minority populations, particularly Korean Americans, are currently lacking.

Evidence of healthcare disparities in Korean Americans has been previously reported (Shin et al., 2018, Jun and Nan, 2017). In an effort to overcome these disparities in Koreans and Korean Americans living in Los Angeles, our study focused on understanding the main obstacles in the way of delivering health messages and screening. We paid special focus on methods that retain the essential elements of original health interventions while also addressing the different linguistic needs and cultural perspectives of the target population. The results of our study report disparities in the receipt of health information and accessibility within a sample of Korean American immigrants.

The goal of this study was to develop tools using our "Korean Community Health Survey" that could potentially capture research survey data. Ultimately, we hope that the tools developed from this project will lead to the development of efficient methods for identifying factors that contribute to lower healthcare awareness and accessibility among Korean Americans in Los Angeles. By doing so, we can establish guidelines to increase health awareness and cancer screening rates, which will eventually reduce cancer risk, in Korean Americans.

\section{Methods}

The study protocol was approved by Cedars-Sinai Medical Center institutional review board (Pro00048053).

In an effort to promote health awareness in Koreans and Korean Americans living in Los Angeles and understand their main health concerns and challenges in receiving proper care, we conducted a survey as part of our initiative step. Our "Korean American Health Survey" was designed for people of Korean descent who are over the age of 18, with no upper limit on age. The survey was administered over a three-week period for the pen and paper survey and ten days for the online version in May 2017. In total, there were 532 participants, of which 421 completed a pen and paper 


\section{International Journal of Innovative Research in Medical Science (IJIRMS) \\ Volume 03 Issue 08 August 2018, ISSN: 2455-8737, Imp. Factor - 4.102 \\ Available online at - $\underline{w w w . i j i r m s . i n}$}

survey and 111 completed the online survey. Aside from general health questions, the surveys were completely anonymous and did not ask for any personal information. Participants were recruited from street sides, local businesses (office and company), community clinics, churches, restaurants, coffee shops, schools et al.

The survey consisted of 13 questions, which took approximately 2 minutes to complete. Participants were asked in-person and selfadministered the in-person survey (Korean or English). The online survey was prepared in English only, due to limitations on the survey platform, Survey Monkey. Information sheets were provided to participants prior to the survey. The Community Advisory board members and study staff helped deliver messages regarding the survey goals, procedures, and methods.

\section{Results and Discussion}

\section{Results from the pen and paper survey format}

In total, 421 participants were selected using a simple random sampling method. A constructed 13-item questionnaire was distributed to all subjects. According to self-reported data, among these 421 participants, $54 \%$ were female and $46 \%$ were male. Most participants (53\%) were aged between 50-64 years old. The next largest age group in the participants $(23 \%)$ was aged 65 years and over, followed by those between 30-39 years old (16\%). A rapid decline in participation was observed in the young adult age group (19-28 years old) and middle-aged group (40-44 and 45-49 years old). The average heights and weights of Korean males and females in Los Angeles were $5 \mathrm{ft} 7 \mathrm{in}, 159 \mathrm{lbs}$, and $5 \mathrm{ft} 3 \mathrm{in}, 121.5$ lbs, respectively.

Most of the participants (58\%) reported either exercising no amount in the past seven days or exercising at least 30 minutes 1-2 days a week. A minority (26\%) reported to exercising at least 30 minutes 3-6 days a week, and the fewest number of participants reported exercising at least 30 minutes every day (16\%). Most of the participants $(89 \%)$ reported not smoking, and a smaller majority $(67 \%)$ stated that they do no consume alcohol. The average number of years that participants have lived in the U.S. varied widely, from less than 5 years to over 36 . Only $10 \%$ were reported to be born in the U.S. Health insurance coverage among participants was diverse: Medicare (20\%), Medi-Cal (23\%), employer-sponsored insurance (25\%), or self-employed insurance (17\%). A small number were reported to be uninsured (11\%).

More than half the participants reported visiting a private doctor's office when sick, needing medical advice, or acquiring a routine check-up. Some (9\%) reported seeking acupuncture when sick. Most participants (24\%) reported that their major concerns were cancer prevention, this was followed by diabetes $(21 \%)$ and heart disease (19\%). Although a majority of participants (84\%) reported having heard of Cedars-Sinai Medical Center, many were not aware of what kinds of treatments and services were available. Roughly half of the participants were interested in being involved in future clinical research.

\section{Results from online survey format}

In total, 111 participants were recruited for the online survey. Approximately $59 \%$ were female and $41 \%$ were male. Most male participants were aged 18-29 (59\%). The second largest age groups were $30-39$ and $40-44(17 \%, 17 \%)$. However, a rapid decline was observed in the age groups $45-49(4 \%)$ and 50-64 (2\%). For females, the majority of participants were from the same age group as males, 18-29 (54\%). Females from age groups 30-39, 40-44, 4549 , and 50-64 were all similar in participation rates; $11 \%, 12 \%$, $11 \%$, and $12 \%$, respectively. The online survey had no participants in the age group of $65+$. The average heights and weight of males and females were $5 \mathrm{ft} 8 \mathrm{in}, 159 \mathrm{lbs}$, and $5 \mathrm{ft} 3 \mathrm{in}, 129 \mathrm{lbs}$, respectively. About $34 \%$ of females and $30 \%$ of males reported walking at least $30 \mathrm{~min} /$ day for 1-2 days/week. More males (46\%) reported walking at least $30 \mathrm{~min} /$ day 3-6 times/week compared to females (34\%). A similar percentage of females and males reported walking every day for at least $30 \mathrm{~min} /$ day; $18 \%$ and $22 \%$, respectively.

A greater percentage of females reported not walking at all for at least $30 \mathrm{~min} /$ day (14\%) compared to males (2\%). Most participants (93\%) did not smoke, and a near majority (45\%) reported not consuming alcohol. The rest (55\%) reported drinking less than 14 drinks/week. Many participants lived in the U.S. for ( $\mathrm{xx}$ ) years on average, and $35 \%$ were born in the U.S. A majority had employersponsored health insurance $(58 \%)$; the remainder reported Medicare (5\%), Medi-Cal (14\%), self-employed insurance (8\%), or other $(7 \%)$. Some were uninsured $(9 \%)$.

The main health concern among this group was back pain (21\%), followed by heart disease (16\%) and cancer prevention $(15 \%)$. Some participants reported being concerned about diabetes (11\%), dementia (6\%), arthritis (6\%), and cancer therapy (2\%). A majority of participants (70\%) reported having heard of Cedars-Sinai Medical Center, but many were unaware of the services provided. A large proportion $(61 \%)$ reported that they did not want to be involved in clinical research.

\section{Paper survey participants walk less than online survey participants}

A majority of paper survey participants did not walk the recommended walking amount; $34 \%$ of females and $30 \%$ of males reported not walking at all for at least $30 \mathrm{~min}$. In contrast, only $14 \%$ of females and $2 \%$ of males in the online survey group reported not walking at all. In general, the online survey group presented a higher rate of exercise; of all online participants, $18 \%$ of female and $22 \%$ of male subjected reported walking at least 30 min every day. Although the number of participants who walked every day was not significantly different, a greater number of online survey participants ( $34 \%$ female and $46 \%$ male) walked at least $30 \mathrm{~min}$ for 3-6 days/week compared to paper survey participants (27\% females and $25 \%$ males). Both surveys exhibited similar patterns for participants who walked at least $30 \mathrm{~min}$ for 1-2 days/week.

\section{Online survey group showed more alcoholic consumption}

Of all paper survey participants, $78 \%$ of females and $54 \%$ of males were non-drinkers. Approximately $22 \%$ of females reported consuming less than 14 drinks/week. Of the male drinkers, $6 \%$ reported being heavy drinkers (more than 14 drinks/week) and $40 \%$ were light drinkers (less than 14 drinks/week). A significantly higher proportion of online survey participants reported higher amounts of drinking. Of the total online survey group, $48 \%$ of females and $41 \%$ of males were non-drinkers. There were less heavy drinkers, but a higher percentage of males (57\%) and females (52\%) were light drinkers (Figure 1). 
International Journal of Innovative Research in Medical Science (IJIRMS)

Volume 03 Issue 08 August 2018, ISSN: 2455-8737, Imp. Factor - 4.102

Available online at - $\underline{w w w . i j i r m s . i n}$

Figure 1: Different patterns of alcohol consumption among participants in the paper vs. online survey.
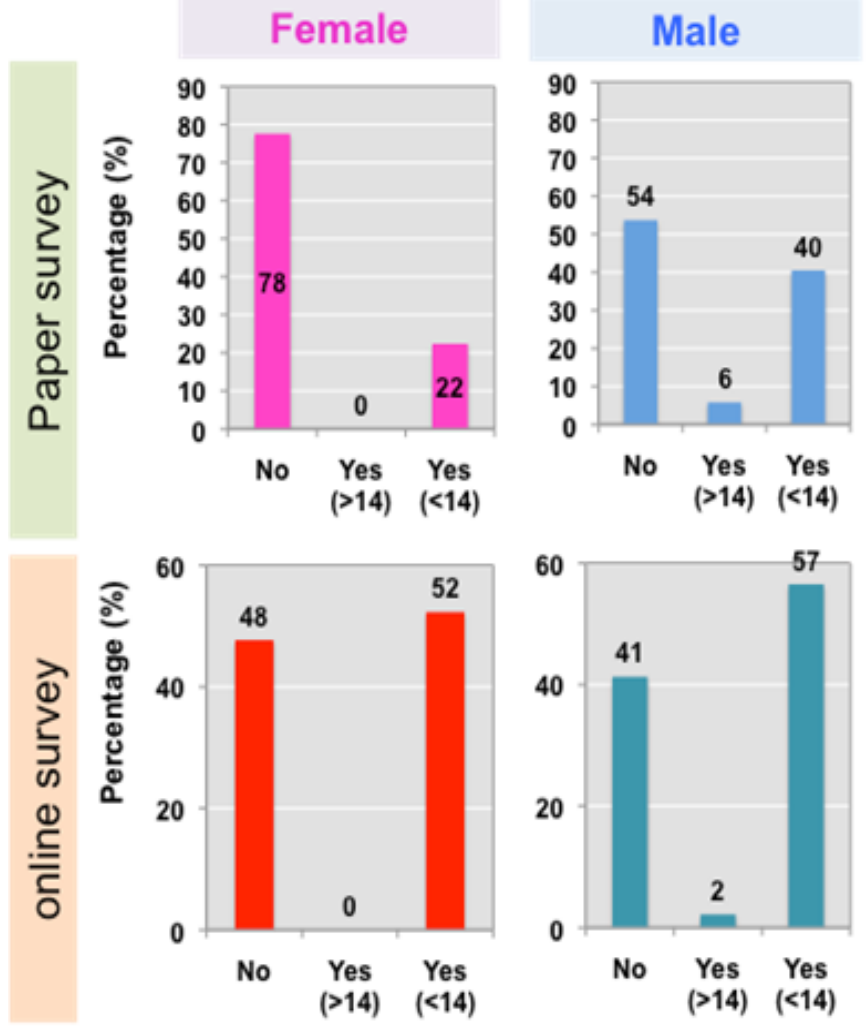

Figure 2: Age distribution of subjects who participated in the paper vs. online survey.

Age
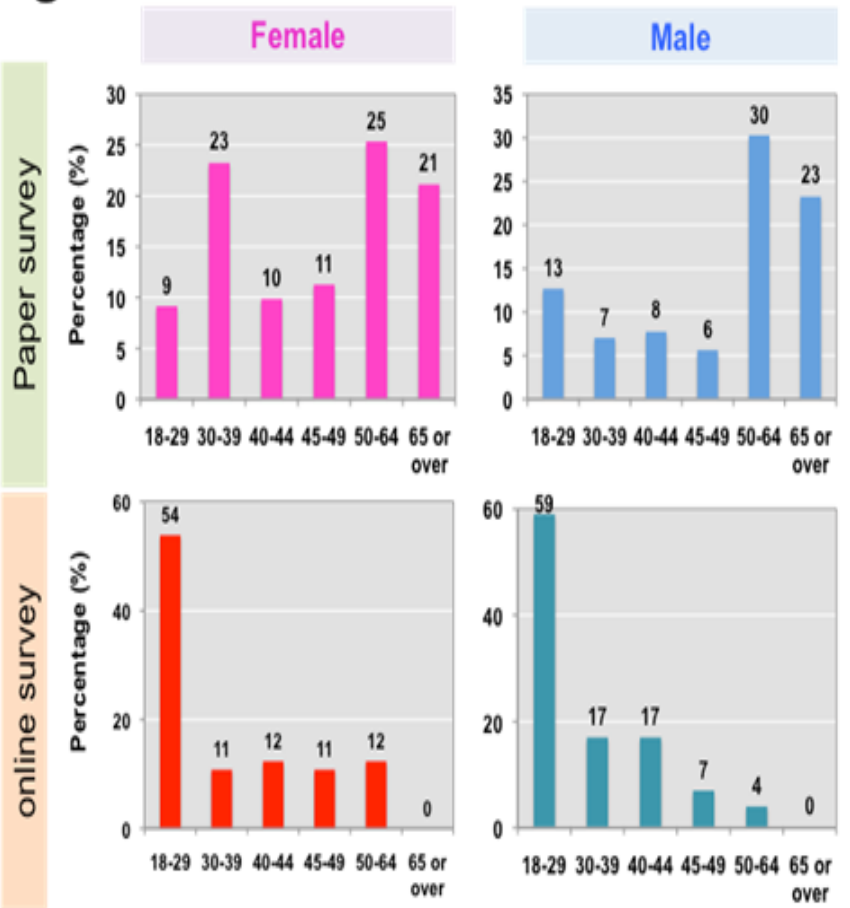

Figure 3: Major health concerns among participants in the paper vs. online survey.

\section{Health concern}

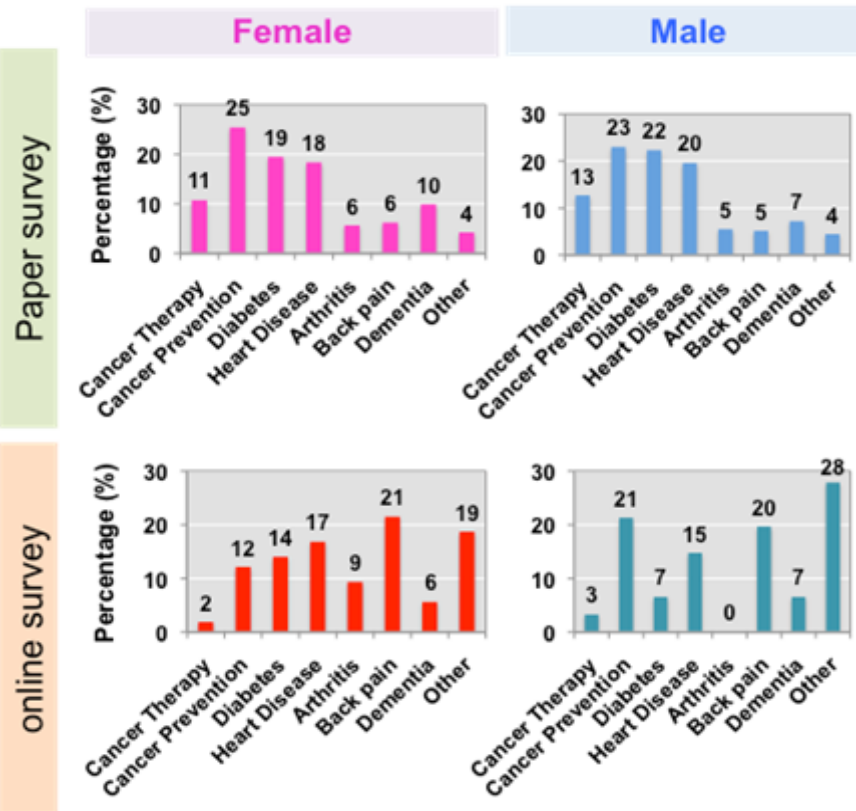

Figure 4: Interest in clinical trials among participants in the paper vs. online survey.

\section{Interest in clinical research}

Female
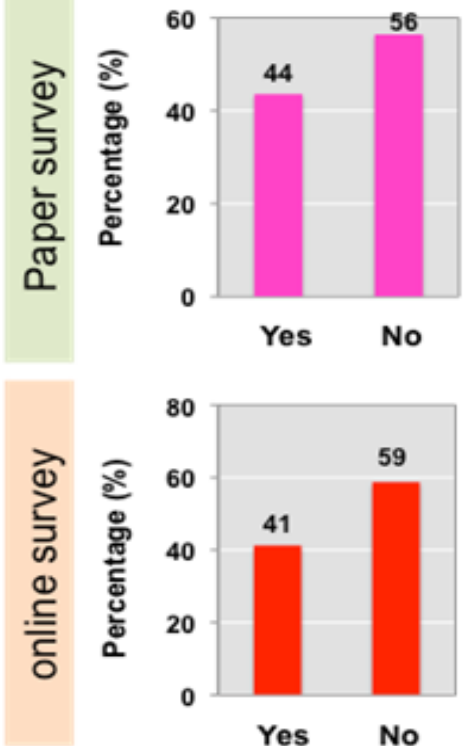

Male
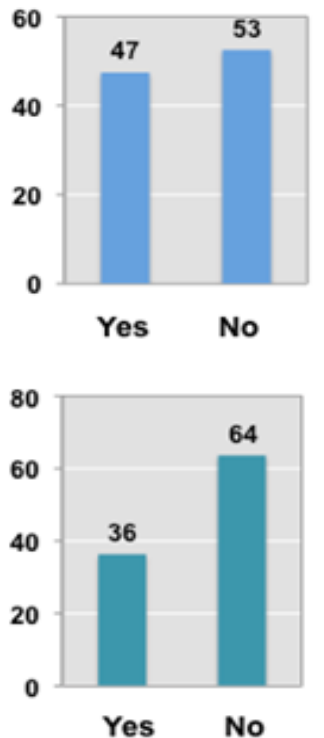

Major health concern of the paper survey group was cancer prevention

One of interesting observations was that age ranges are dramatically different between pen and paper vs. online surveys (Figure 2). Among some common health problems, cancer prevention was the major health concern ( $25 \%$ of all participants) in paper survey. It was followed by diabetes (21\%) and heart disease (19\%). In contrast, participants in the online survey groups reported back pain as their major concern $(21 \%)$, followed by heart disease (16\%) and cancer prevention (15\%) (Figure 3). 
Interestingly, both surveys showed that a majority of participants were not interested in clinical research; $55 \%$ of paper survey participants and $61 \%$ of online survey participants reported not being interested in clinical research (Figure 4). These factors should be carefully considered for future studies with Korean American immigrants (Lee, 2015).

In addition, this study showed that we may not get a strong response from males over 45 years old if using an online survey. We found that subjects who completed the paper survey did not walk enough; about $30 \%$ of males and $34 \%$ of females. The online survey group was generally younger and showed more alcohol consumption.

It should be noted that paper survey participants preferred the Korean version of the survey, regardless of English-speaking skills. It suggests the importance of language and comfort when obtaining health messages. Even those who did not have any language barriers thought that a culturally comfortable environment is critical for efficient message delivery. These findings suggest that language-based and culturally-appropriate questionnaires will enhance the effectiveness of health information delivery and survey-based future studies.

\section{Disclosure of Potential Conflicts of Interest}

No potential conflicts of interest were disclosed.

\section{Acknowledgements}

We thank the leaders of the community-based organizations and churches, and the Korean Americans who participated in the study. The author acknowledges support from National Institutes of Health grants (1U01DK103260, 1R01DK100974, U24 DK097154, NIH NCATS UCLA CTSI UL1TR000124), Department of Defense grants (W81XWH-15-1-0415), Centers for Disease Controls and Prevention (1U01DP006079), IMAGINE NO IC Research Grant, the Steven Spielberg Discovery Fund in Prostate Cancer Research Career Development Award, and the U.S.-Egypt Science and Technology Development Fund by the National Academies of Sciences, Engineering, and Medicine (to J.K.). J.K. is former recipient of Interstitial Cystitis Association Pilot Grant, a Fishbein Family IC Research Grant, New York Academy of Medicine, and Boston Children's Hospital Faculty Development. The funders had no role in the design, data collection and analysis, decision to publish, or preparation of the manuscript.

\section{Reference}

[1] BARRERA, M., JR., CASTRO, F. G., STRYCKER, L. A. \& TOOBERT, D. J. 2013. Cultural adaptations of behavioral health interventions: a progress report. J Consult Clin Psychol, 81, 196-205.

[2] JUN, J. \& NAN, X. 2017. Determinants of Cancer Screening Disparities Among Asian Americans: A Systematic Review of Public Health Surveys. J Cancer Educ.

[3] LEE, S. Y. 2015. Cultural Factors Associated with Breast and Cervical Cancer Screening in Korean American Women in the US: An Integrative Literature Review. Asian Nurs Res (Korean Soc Nurs Sci), 9, 81-90.
[4] SHIN, C. N., KELLER, C. \& SIM, J. 2018. Cultural Factors relevant to Korean Americans in Health Research: A Systematic Review. J Community Health, $43,421-432$.

\section{IICorrespondence Author -}

Jayoung Kim, PhD.

Cedars-Sinai Medical Center, Departments of Surgery and Biomedical Sciences

8700 Beverly Blvd., Los Angeles, CA 90048

Tel: +1-310-423-7168

Fax:+1-310-967-3809

E-mail: Jayoung. Kim [at] cshs.org 